\title{
Correlation analysis of alveolar bone loss in buccal/palatal and proximal surfaces in rats
}

\section{Carolina Barrera de Azambuja Juliano Cavagni Marcius Comparsi Wagner Eduardo José Gaio Cassiano Kuchenbecker Rösing}

Department of Periodontology, School of Dentistry, Univ Federal do Rio Grande do Sul - UFRGS, Porto Alegre, RS, Brazil.

Declaration of Interests: The authors certify that they have no commercial or associative interest that represents a conflict of interest in connection with the manuscript.

Corresponding Author:

Juliano Cavagni

E-mail: jcavagni@hotmail.com

Submitted: May 08, 2012

Accepted for publication: Sep 06, 2012

Last revision: Sep 25, 2012

\begin{abstract}
The aim was to correlate alveolar bone loss in the buccal/palatal and the mesial/distal surfaces of upper molars in rats. Thirty-three, 60-day-old, male Wistar rats were divided in two groups, one treated with alcohol and the other not treated with alcohol. All rats received silk ligatures on the right upper second molars for 4 weeks. The rats were then euthanized and their maxillae were split and defleshed with sodium hypochlorite (9\%). The cemento-enamel junction (CEJ) was stained with $1 \%$ methylene blue and the alveolar bone loss in the buccal/palatal surfaces was measured linearly in 5 points on standardized digital photographs. Measurement of the proximal sites was performed by sectioning the hemimaxillae, restaining the CEJ and measuring the alveolar bone loss linearly in 3 points. A calibrated and blinded examiner performed all the measurements. Intraclass Correlation Coefficient revealed values of 0.96 and 0.89 for buccal/lingual and proximal surfaces, respectively. The Pearson Correlation Coefficient ( $\mathrm{r}$ ) between measurements in buccal/ palatal and proximal surfaces was 0.35 and 0.05 for the group treated with alcohol, with and without ligatures, respectively. The best correlations between buccal/palatal and proximal surfaces were observed in animals not treated with alcohol, in sites both with and without ligatures $(r=0.59$ and 0.65 , respectively). A positive correlation was found between alveolar bone loss in buccal/palatal and proximal surfaces. The correlation is stronger in animals that were not treated with alcohol, in sites without ligatures. Areas with and without ligature-induced periodontal destruction allow detection of alveolar bone loss in buccal/palatal and proximal surfaces.
\end{abstract}

Descriptors: Alveolar Bone Loss; Animals; Periodontitis.

\section{Introduction}

Periodontitis is a disease characterized by periodontal tissue destruction caused by the host response against bacteria and their toxins. The absence of treatment can lead to the loss of the tooth affected by the disease. $^{1}$

In the study of the pathogenesis of periodontitis, there are strong limitations in the use of models involving humans for logistic and ethical reasons. Because of these limitations, animal models have been widely used in literature. Among the experimental models for destructive periodontal disease study, monkeys, dogs and small animals have been used. Rats 
are one of the most frequently used models, since they share similarities with humans. These similarities apply to periodontal anatomy, development and compositions of biofilms, histopathology and immunobiology of periodontal lesions. ${ }^{2}$

Following the experimental periods, it is important to quantify the destruction, in order to understand the effect of the factor studied in the experimental development of the disease. Animal models make it possible to make different morphological measurements, which of course is not possible with human models. These measurements enable metrical quantification of the result and of the inflammatory process. For this purpose, different methods for measuring alveolar bone loss in rats have been proposed in the literature, such as histometric, ${ }^{3}$ morphometric $^{4-6}$ conventional radiographic measurements $^{7}$ and computed tomography. ${ }^{8,9}$

Different studies have sought to demonstrate the ability to measure periodontal destruction by different methods and effectively quantify the effect caused by the experimental model of periodontal disease. Fernandes et al. ${ }^{10}$ compared the histometric and morphometric methods and found that both are capable of detecting alveolar bone loss in rats. $\mathrm{Li}$ and $A_{m a r}{ }^{11}$ likewise compared the different methods-morphometric isolated, histometric and morphometric associated and micro-computed tomography-in 100 male rats, and their findings showed that all the methods are accurate for quantification of alveolar bone loss.

In addition to the ability to detect the destruction, there is the issue, raised in the literature, of the best site and the best way to measure the observed effects. For example, there are studies comparing the morphometric method with area versus linear measurements. ${ }^{12-14}$ However, these two systems allow evaluation of alveolar bone loss in lingual/ palatal and buccal surfaces only. To the best of our knowledge, studies evaluating proximal bone loss are inexistent in the literature to date.

Thus, the aim of this study was to correlate alveolar bone loss in buccal/palatal and proximal surfaces of upper molars in Wistar rats.

\section{Methodology Sample}

The data used in the present study included a subset of the project entitled "Effect of the chemical dependence of $15 \%$ ethanol on ligature-induced alveolar bone loss in Wistar rats." Thirty-three male, 60-day-old Wistar rats were used, 15 not treated and 18 treated with alcohol. A randomized, blind and controlled animal model study was performed. The project compared alcohol treated animals during the whole study period with animals not exposed to alcohol. It was a methodological study that compared intragroup measurements of alveolar bone loss, without taking alcohol exposure specifically into consideration. All rats received 4-0 silk ligatures (Ethicon, Johnson \& Johnson ${ }^{\circledR}$, São Paulo, Brazil) for induction of alveolar bone loss around the upper right second molars. The left side remained as intragroup control. In all, 66 hemimaxillae were used (33 with ligature and 33 without). The induction of alveolar bone loss occurred during a period of four weeks.

During the entire experimental period, the rats remained at the Center of Reproduction and Experimentation of Laboratory Animals (CREAL), at the Federal University of Rio Grande do Sul. Animals were kept in boxes, in rooms with a controlled temperature $\left(20^{\circ} \mathrm{C}\right)$ and in a light/dark cycle of 12 hours. The animals received standard diet ad libitum during the study.

The study protocol was submitted and approved by the Research Ethics Committee of the Federal University of Rio Grande do Sul. The protocol complies with the regulations set down by the Universal Declaration of Animal Rights (UNESCO - January 27, 1978) and the International Ethical Guidelines for Biomedical Research Involving Animals (Council for International Organizations of Medical Sciences - CIOMS).

\section{Specimen preparation}

The animals were euthanized by decapitation. Specimen preparation and morphometric analysis were conducted at the Laboratory of Periodontology at the Federal University of Rio Grande do Sul, 
and followed the method proposed by Fernandes et al. ${ }^{10}$ Soft tissue was removed by defleshing the maxillae with sodium hypochlorite solution (9\%) (Mazarollo, Gravataí, Brazil) for five hours, and then removing the remaining tissues manually. The specimens were then washed and dried. Specimens were stained with $1 \%$ methylene blue for one minute for better visualization of the CEJ. Pictures were taken using a 6.1 megapixel digital camera (Nikon ${ }^{\circledR}$ Coolpix, Ayutthaya, Thailand) coupled to a tripod and equipped with $100 \mathrm{~mm}$ macro-lenses with minimal focal distance. The hemimaxillae were placed with the occlusal surface parallel to the floor. ${ }^{4,5,10,6,13}$

\section{Buccal/palatal morphometric analysis}

The buccal/palatal morphometric analysis was performed by evaluating standard digital photographs. An examiner, calibrated and blinded to the experimental groups, performed the measurements of the linear distances of the CEJ to the bone crest, using Image Tool 3.0. software (UTHSCPA, San Antonio, USA). Five measurements were performed on each surface (two on the distal root, two on the mesial root and one on the furcation). The measurements in pixels were then converted into millimeters using the markings of the endodontic ruler to which the hemimaxillae were attached as reference. Each hemimaxillae generated a mean value for analysis at a later time. Figure 1 shows the specimen and the measurements made on the buccal surface in the site with no ligature.

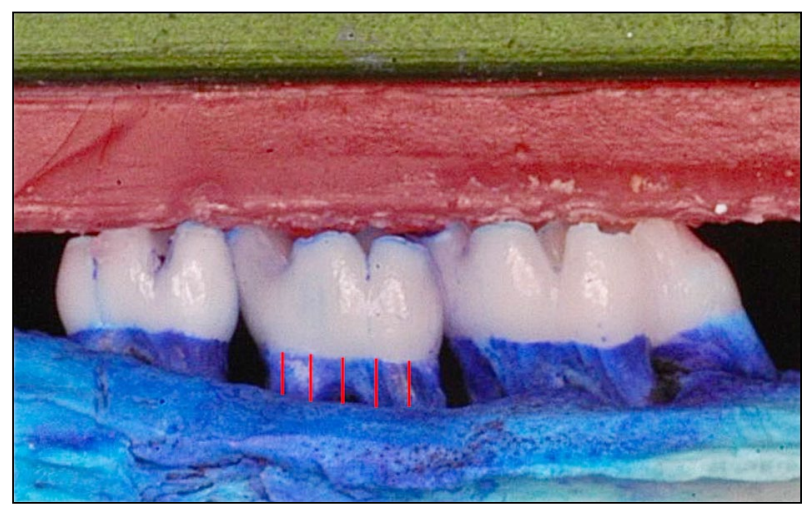

Figure 1 - Linear measurements made on digital photographs of the buccal surface of hemimaxillae attached to an endodontic ruler in the site without ligature.

\section{Proximal site morphometric analysis}

After morphometric analysis of buccal/palatal surfaces, the hemimaxillae were cut with hand pieces and diamond burs under refrigeration, in mesial and distal aspects of the second molar, preserving the bone crest. The CEJ was restained with $1 \%$ methylene blue, and alveolar bone loss was evaluated in three regions (buccal root, center of the proximal surface and palatal root) of each proximal surface (mesial and distal). Before cutting, a reference mark was made in order to identify the mesial surface. Each hemimaxillae generated a mean value for later analysis. Figure 2 shows the measurements for the site with ligature on the proximal aspects.

\section{Blinding}

Examiner blinding was assured before the morphometric analysis. After the photographs were taken, they were all codified by an external examiner, so that the investigators would not know to which group each specimen belonged when analyzed. After measurement was concluded, the code was broken and the photographs were renamed according to the respective group.

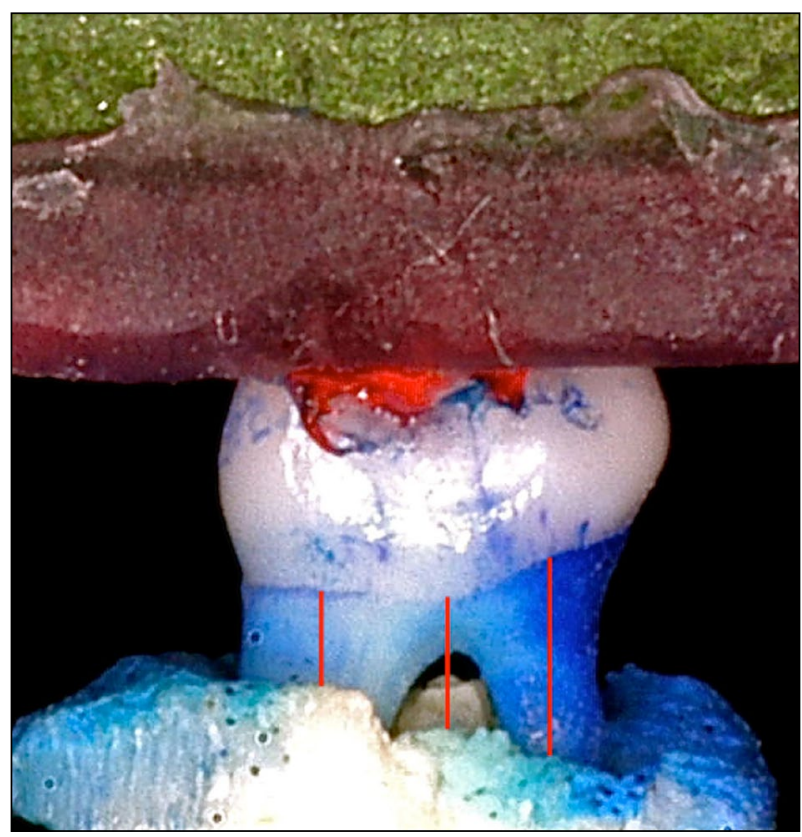

Figure 2 - Linear measurements made on digital photographs of the proximal surface of hemimaxillae attached to an endodontic ruler in the site with ligature. 


\section{Reproducibility}

Reproducibility was checked by testing and retesting, with a one-week interval between measurements. Scatter Graphics and Intraclass Correlation Coefficient (ICC) were used to evaluate reproducibility. The ICC of these measurements was 0.96 and 0.89 for buccal/palatal and proximal surfaces, respectively. These values are considered excellent by the literature.

\section{Data analysis}

Parametric tests were used to determine possible correlations and statistical differences between the experimental groups, insofar as the data presented normal distribution. Pearson Correlation Coefficient $(r)$ was used to determine the grade of correlation between measurements of buccal/palatal and proximal surfaces. Cutoff points were $>0.70$ : strong correlation; 0.30 to 0.70 : moderate correlation; $<0.30$ : weak correlation. All data analyses were performed using Stata $^{\oplus} 10.1$ for Macintosh software (Stata Corporation, College Station, USA).

\section{Results}

The results of the present study are shown in Figures 3-6. Correlations were assessed separately between buccal/palatal and proximal sites for teeth with and without ligature in the hemimaxillae of rats treated or not treated with alcohol.

The correlation between the variables presented

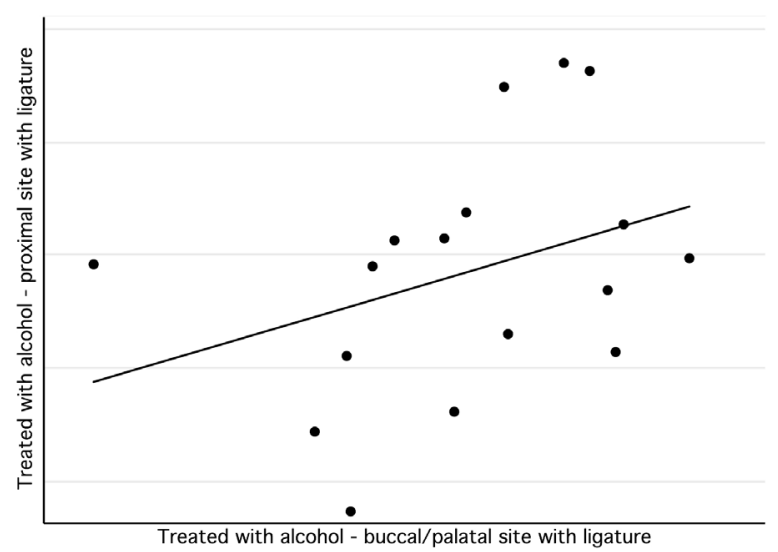

Figure 3 - Scatter plot correlating buccal/palatal and proximal surfaces in the group treated with alcohol and with ligature. a positive/ascendant direction (Figures 3-6) for all comparisons. However, there were different values for the Pearson Correlation Coefficient, depending on the comparisons. In general, Figures 3, 5 and 6 presented a moderate correlation, whereas Figure 4 presented a low correlation. The Pearson Correlation Coefficient between measurements of buccal/ palatal and proximal surfaces was 0.35 and 0.05 in the groups treated with alcohol, in sites with or without ligature, respectively. When groups that were not treated with alcohol are considered, higher correlations were observed between measurements of buccal/palatal and proximal surfaces, with an $r=0.59$ and $r=0.65$ in sites with and without ligature, respectively.

\section{Discussion}

The objective of the present study was to correlate measurements of ligature-induced alveolar bone loss in Wistar rats, in buccal/palatal and proximal surfaces, using specimens from a controlled experimental study. It was observed that the measurements for animals not treated with alcohol presented good correlation. It is important to emphasize that $100 \%$ of the specimens were evaluated, with no losses. The CEJ was evident in all specimens, both in the buc$\mathrm{cal} /$ palatal and in the proximal surfaces. This indicates that it is possible and reliable to prepare the specimens without losing information. There were no difficulties in analyzing the proximal surfaces.

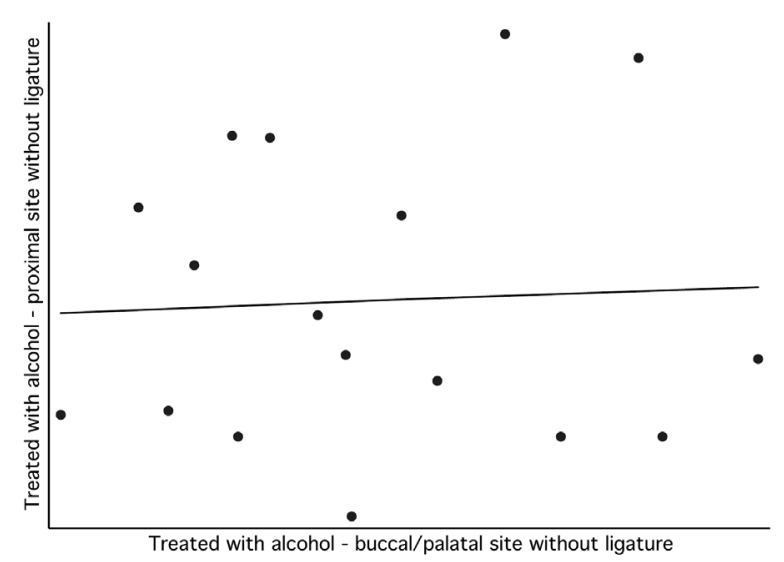

Figure 4 - Scatter plot correlating buccal/palatal and proximal surfaces in the group treated with alcohol and without ligature. 




Figure 5 - Scatter graph correlating buccal/palatal and proximal surfaces in the group not treated with alcohol and with ligature.

Experimental studies in animals are fundamental in the context of etiopathogenic research of the morbid processes. This situation relies on the idea that it is important to know the different interferences that may occur in the causal chain of diseases. These studies cannot be performed in humans because of ethical reasons, and also because it is impossible to isolate the variables, which is possible to do in animal models. ${ }^{15,2}$ Nonetheless, it is important to clarify that data obtained in animals represent a step toward gaining the needed knowledge, but must not be directly extrapolated to clinical situations. ${ }^{16}$

One of the most important aspects to be highlighted in the present study is that the potential benefits of understanding the pathogenesis of periodontal destruction in rats can transcend dentistry. In the health sciences, it is very difficult to quantify inflammation and its effects with objective and reproducible measurements. This is done today mostly by analyzing the quantity and intensity of inflammatory cells in different manners. In the specific case of periodontal disease, the inflammatory process leads to tissue destruction, an effect that is quantifiable by objective criteria. Therefore, the experimental models of periodontal disease induction in animals have the potential to be used for studying other chronic inflammatory processes, if what is intended is to understand the chain of events and to isolate variables. These measurements are reproducible and, in the present study, the Intraclass Correlation Coefficients

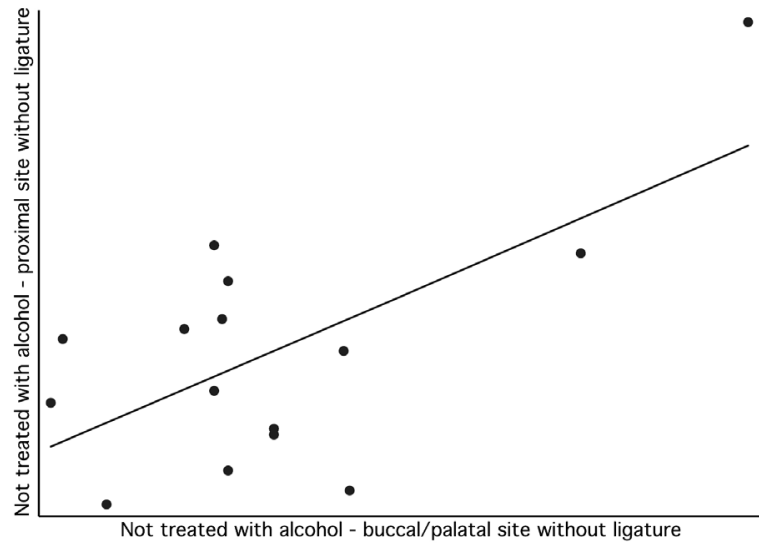

Figure 6 - Scatter graph correlating buccal/palatal and proximal surfaces in the group not treated with alcohol and without ligature.

for buccal/palatal surfaces and proximal surfaces were about 0.9 , a figure considered excellent. Experimental models such as that used in the present study are useful, because they allow adequate examiner blinding and repeated analyses. This increases the quality of the information generated.

It is also important to highlight that the way alveolar bone loss was measured in the present study is also at issue. It is possible to measure periodontal destruction by histometric, morphometric and also image examinations. Studies published by Fernandes et al., ${ }^{10}$ Park et al. ${ }^{8}$ and Wilensky et al. ${ }^{9}$ demonstrated that these three outcome-measuring methodologies are possible and capable of detecting differences among the factors studied.

Different studies have addressed the measurement issue in distinct ways. For instance, some studies have measured the destruction in the furcation area, others in proximal sites, some in buccal and lingual areas, others with only one measurement, or repeated measurements, etc. ${ }^{14,17-24}$ To the best of our knowledge, there are no studies evaluating ligatureinduced alveolar bone loss in proximal and buccal/ palatal surfaces in rats. Although ligature-induced alveolar bone loss measurement in proximal surfaces may be difficult to perform in morphometric analyses, the focus of the present study was to examine differences in the prevalence and extension of periodontal disease in proximal surfaces in humans, which could also occur in animals. ${ }^{25}$ 
The results obtained in the present study showed a positive correlation between the measurements of buccal/palatal and proximal surfaces in groups not treated with alcohol, in sites both with and without ligature. In the group treated with alcohol, these correlations were less intense. This may be explained by the fact that other factors may interfere in the outcomes more easily when there is a modifier. Interestingly, there is a study that has shown differences between the groups only in the site without ligature. ${ }^{6}$ This has been examined in the research on etiopathogenesis of periodontal diseases in animal models, and explanations are still scarce. In the present study, an $r=0.59$ was obtained for the group not treated with alcohol, in the site with ligature, and a value of $r=0.65$ was obtained for the site without ligature. The higher value obtained for this correlation coefficient corroborates that the chances of higher associations between the measurements increase when there are no interferences.

The results of the present study are challenging

\section{References}

1. Page RC, Kornman KS. The pathogenesis of human periodontitis: an introduction. Periodontol 2000. 1997 Jun;14(1):9-11.

2. Susin C, Rösing CK. O rato como modelo para o estudo das repercussões do estresse nas doenças periodontais. Rev Periodontia. 2002;13(6):5-10.

3. Semenoff TA, Semenoff-Segundo A, Bosco AF, Nagata MJ, Garcia VG, Biasoli ER. Histometric analysis of ligatureinduced periodontitis in rats: a comparison of histological section planes. J Appl Oral Sci. 2008 Jul-Aug;16(4):251-6.

4. Cavagni J, Soletti AC, Gaio EJ, Rosing CK. The effect of dexamethasone in the pathogenesis of ligature-induced periodontal disease in Wistar rats. Braz Oral Res. 2005 OctDec;19(4):290-4.

5. Daudt LD, Cavagni J, Gaio EJ, Souza A, Torres IL, Ferreira $\mathrm{MB}$, et al. Effect of inhaled corticosteroid on TNF-alpha production and alveolar bone loss in Wistar rats. Arch Oral Biol. 2011 Nov;56(11):1398-403.

6. Liberman DN, Pilau RM, Gaio EJ, Orlandini LF, Rosing CK. Low concentration alcohol intake may inhibit spontaneous alveolar bone loss in Wistar rats. Arch Oral Biol. 2011 Feb;56(2):109-13.

7. Semenoff Segundo A, Semenoff TA, Borges AH, Pedro FL, Sakai VT. Methodological model of chronic stress associated with ligature-induced periodontitis in rats: a radiographic study. Braz Oral Res. 2010 Oct-Dec;24(4):455-9. and show that it is possible to detect destruction of the periodontium in buccal/palatal and also in proximal surfaces after an experimental period. Thus, it is optional where the researcher conducts the measurements; the best place would be that which is most suitable to posit the hypothesis of his research. In addition, the results show that it is not important to conduct two distinct methods, i.e., analysis in buccal/palatal and proximal surfaces, in order to evaluate alveolar bone loss; either one or the other may be conducted.

\section{Conclusion}

In models of periodontal destruction induced by ligature, there is correlation in alveolar bone loss between buccal/palatal and proximal surfaces. The correlation is stronger in animals not treated with alcohol. In areas with and without ligature-induced periodontal disease, it is possible to detect alveolar bone loss in buccal/palatal and proximal surfaces.

8. Park CH, Abramson ZR, Taba Jr M, Jin Q, Chang J, Kreider JM, et al. Three-dimensional micro-computed tomographic imaging of alveolar bone in experimental bone loss or repair. J Periodontol. 2007 Feb;78(2):273-81.

9. Wilensky A, Gabet Y, Yumoto H, Houri-Haddad Y, Shapira L. Three-dimensional quantification of alveolar bone loss in Porphyromonas gingivalis-infected mice using micro-computed tomography. J Periodontol. 2005 Aug;76(8):1282-6.

10. Fernandes MI, Gaio EJ, Oppermann RV, Rados PV, Rosing CK. Comparison of histometric and morphometric analyses of bone height in ligature-induced periodontitis in rats. Braz Oral Res. 2007 Jul-Sep;21(3):216-21.

11. Li CH, Amar S. Morphometric, histomorphometric, and microcomputed tomographic analysis of periodontal inflammatory lesions in a murine model. J Periodontol. 2007 Jun;78(6):1120-8.

12. Kuhr A, Popa-Wagner A, Schmoll H, Schwahn C, Kocher T. Observations on experimental marginal periodontitis in rats. J Periodontal Res. 2004 Apr;39(2):101-6.

13. Liberman DN, Pilau RM, Orlandini LF, Gaio EJ, Rosing CK. Comparison of two methods for alveolar bone loss measurement in an experimental periodontal disease model in rats. Braz Oral Res. 2011 Jan-Feb;25(1):80-4.

14. Souza DM, Prado FA, Prado MA, Rocha RF, Carvalho YR. Evaluation of two morphometric methods of bone loss per- 
centages caused by periodontitis in rats in different locations. J Appl Oral Sci. 2010 Sep-Oct;18(5):493-7.

15. Graves DT, Fine D, Teng YT, Van Dyke TE, Hajishengallis G. The use of rodent models to investigate host-bacteria interactions related to periodontal diseases. J Clin Periodontol. 2008 Feb;35(2):89-105.

16. Oz HS, Puleo DA. Animal models for periodontal disease. J Biomed Biotechnol. 2011;2011:754857. Epub 2011 Feb 10.

17. Barros SP, Silva MA, Somerman MJ, Nociti Jr FH. Parathyroid hormone protects against periodontitis-associated bone loss. J Dent Res. 2003 Oct;82(10):791-5.

18. Bjornsson MJ, Velschow S, Stoltze K, Havemose-Poulsen A, Schou S, Holmstrup P. The influence of diet consistence, drinking water and bedding on periodontal disease in SpragueDawley rats. J Periodontal Res. 2003 Dec;38(6):543-50.

19. Cesar-Neto JB, Benatti BB, Haiter Neto F, Sallum AW, Sallum EA, Nociti Jr FH. Smoking cessation may present a positive impact on mandibular bone quality and periodontitis-related bone loss: a study in rats. J Periodontol. 2005 Apr;76(4):520-5.

20. Duarte PM, Goncalves PF, Sallum AW, Sallum EA, Casati MZ, Nociti Jr FH. Effect of an estrogen-deficient state and its therapy on bone loss resulting from an experimental periodontitis in rats. J Periodontal Res. 2004 Apr;39(2):107-10.

21. Fine DH, Schreiner H, Nasri-Heir C, Greenberg B, Jiang S, Markowitz K, et al. An improved cost-effective, reproducible method for evaluation of bone loss in a rodent model. J Clin Periodontol. 2009 Feb;36(2):106-13.

22. Mitsuta T, Horiuchi H, Shinoda H. Effects of topical administration of clodronate on alveolar bone resorption in rats with experimental periodontitis. J Periodontol. 2002 May;73(5):479-86.

23. Niikura K, Takeshita N, Chida N. A novel inhibitor of vacuolar ATPase, FR202126, prevents alveolar bone destruction in experimental periodontitis in rats. J Toxicol Sci. 2005 Dec;30(4):297-304.

24. Vacas MI, Amer M, Chiarenza AP, Luchelli MA, Mandalunis PM, Elverdin JC. Influence of submandibulectomy on alveolar bone loss in rats. J Periodontol. 2008 Jun;79(6):1075-80.

25. Albandar JM, Brunelle JA, Kingman A. Destructive periodontal disease in adults 30 years of age and older in the United States, 1988-1994. J Periodontol. 1999 Jan;70(1):13-29. 